\title{
Chapter 16 \\ Enhancement of Transmutation of Minor Actinides by Hydride Target
}

\author{
Kenji Konashi and Tsugio Yokoyama
}

\begin{abstract}
A hydride target including minor actinides (MA) is able to enhance the transmutation rate in a fast breeding reactor (FBR) without degradation of core safety characters. Fast neutrons generated in the core region of the FBR are moderated in the MA-hydride target assemblies and then efficiently absorbed by MA. The MA-hydride target pin has been designed in the light of recent research of hydride materials. This chapter shows the feasibility of MA transmutation by an existing reactor, Monju.
\end{abstract}

Keywords Fast breeder reactor $\bullet$ Hydride $\bullet$ Minor actinide

\subsection{Introduction}

High-level wastes generated after reprocessing spent nuclear fuels include longlived radioactive nuclides of minor actinides (MA), such as ${ }^{237} \mathrm{~Np},{ }^{241} \mathrm{Am},{ }^{243} \mathrm{Am}$, and ${ }^{244} \mathrm{Cm}$. A currently available method for the final disposal of the high-level wastes is to vitrify them under rigid control, to store them in monitored locations until the radiation decays to allowable levels, and then to dispose them underground.

The transmutation of MA by the fast breeder reactor (FBR) has been intensively studied to reduce radioactivity of the wastes [1]. Transmutation rate, which is one of the most important factors for transmutation methods, is determined by values of neutron flux and nuclear reaction cross section as follows:

$$
\lambda=\int \Phi_{n}(E) \sigma_{n}(E) \mathrm{d} E
$$

K. Konashi $(\bowtie)$

Institute for Materials Research, Tohoku University, Oarai, Ibaraki-ken 311-1313, Japan

e-mail: konashi@imr.tohoku.ac.jp

T. Yokoyama

Toshiba Nuclear Engineering Services Corporation, 8 Shinsugita, Isogo-ku, Yokohama

235-8523, Japan 
FBRs provide high fast neutron flux, wherein the neutron reaction cross sections are small compared with those in the thermal energy region. Moderation of fast neutrons by hydride materials was considered to increase the transmutation rate [2-5]. In this chapter, enhancement of transmutation of MA by an MA-hydride target is studied. Target assemblies containing MA-hydrides are placed in the radial blanket region. Fast neutrons generated in the core region are moderated in the hydride target assembly and then produce high flux of thermal neutrons, which have large nuclear reaction cross sections to actinides. The MA-hydride target also has another advantage to load MA to limited space. The target of (MA, $\mathrm{Zr}) \mathrm{H}_{x}$ increases mass of MA and hydrogen density in the blanket region compared with MA and $\mathrm{ZrH}_{1.6}$ loaded separately [4, 5].

Hydride fuels have been used in TRIGA reactors of General Atomics (GA) for many years [6]. On the other hand, hydride materials do not have much history of use in FBRs. Recently, a control rod of FBR with hafnium (Hf)-hydride has been studied [7]. In this chapter, the MA-hydride target pin was designed using experimental data of Hf-hydride.

\subsection{Design of MA-Hydride Target}

The TRIGA fuel consists of a U-metal phase and a Zr-hydride phase at high temperature in the reactor. The MA-hydrides are stable at high temperature [8]. The phase relationship of the U-Th-Zr hydride has been studied, considering Th as a surrogate of MA. Figure 16.1 shows the microstructure of $\mathrm{UTh}_{4} \mathrm{Zr}_{10} \mathrm{H}_{24}$ : black areas are $\mathrm{Zr}$ hydride, gray region is $\mathrm{ThZr}_{2} \mathrm{H}_{x}$, and white areas are uranium metal. The thermodynamic analysis shows that the MA-hydride consists of MA-hydride, MA-Zr-hydride, and Zr-hydride (Fig. 16.1).

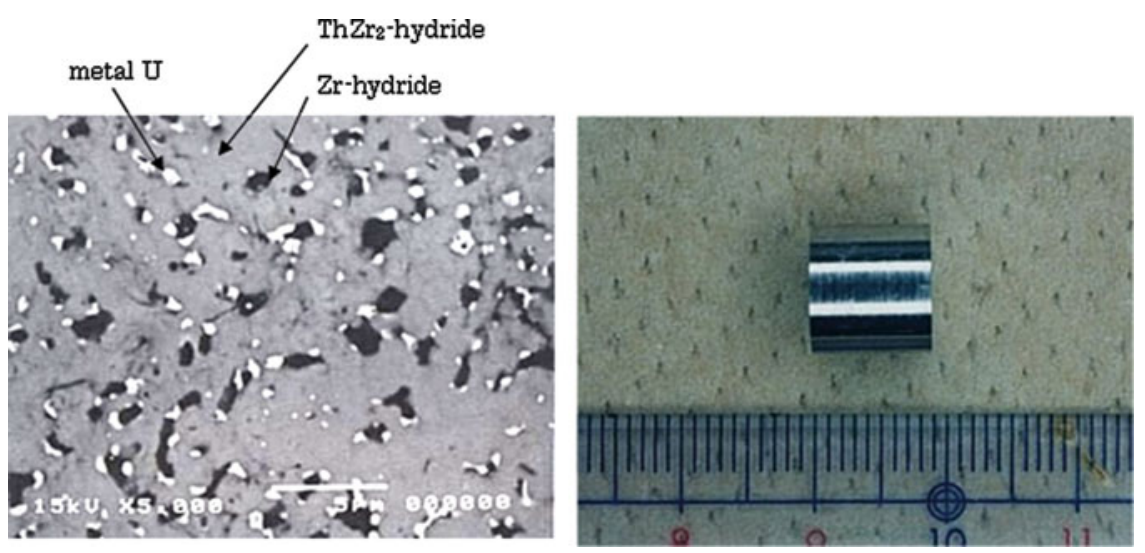

Fig. 16.1 Pellet of $(\mathrm{U}, \mathrm{Th}, \mathrm{Zr}) \mathrm{H}_{x}$ and microstructure 
Fig. 16.2 MA-hydride target pin

For the application of Hf-hydride to neutron absorber material in FBR [3], the Hf-hydride pin has been developed. The fabrication of sodium-bonded Hf-hydride pins has been demonstrated. The pins were successfully irradiated in BOR-60 for 1 year [7]. The MA-target pin was designed based on the foregoing experiences. Figure 16.2 shows a target pin that includes MA-hydride ( $\left.\mathrm{MA}, \mathrm{Zr}) \mathrm{H}_{1.6}\right)$ pellets. The gap of the MA hydride pellet-stainless steel cladding was filled with liquid sodium to keep the temperature of the pellets low. The results of irradiation experiments show that the sodium also reduces loss of hydrogen from the hydride pin.

\subsection{Design of Core with MA-Hydride Target}

Table 16.1 and Fig. 16.3 show the core specification and layout with hydride targets. The core layout is based on the Japanese prototype fast reactor Monju. The thermal power is $714 \mathrm{MWt}$, the diameter of the active core is about $1,800 \mathrm{~mm}$, and the height is $930 \mathrm{~mm}$. The 54 hydride MA-hydride target assemblies are located at the inner most row in the three radial blanket rows. Each assembly contains 61 MA-hydride target pins, where the diameter of the pellets is set at $10.4 \mathrm{~mm}$ and the stack length is $930 \mathrm{~mm}$. The ratio of $\mathrm{H} / \mathrm{M}(\mathrm{M}=\mathrm{MA}+\mathrm{Zr})$ is considered to be 1.6. The composition of MA is assumed that derived from the typical large LWR discharged fuel, that is, ${ }^{237} \mathrm{~Np} /{ }^{241} \mathrm{Am} /{ }^{242 \mathrm{~m}} \mathrm{Am} /{ }^{243} \mathrm{Am} /{ }^{243} \mathrm{Cm} /{ }^{244} \mathrm{Cm} /{ }^{245} \mathrm{Cm} /{ }^{246}$ $\mathrm{Cm}=0.5200 / 0.2493 / 0.0010 / 0.1663 / 0.0006 / 0.0592 / 0.0031 / 0.0006$. 
Table 16.1 Major core specifications for minor actinides (MA) transmutation

\begin{tabular}{l|l}
\hline Reactor type & Fast breeder reactor \\
\hline Cooling system & Sodium cooled (loop-type) \\
\hline Thermal output & $714 \mathrm{MW}$ \\
\hline Electrical output & $280 \mathrm{MW}$ \\
\hline Fuel & Mixed oxide \\
\hline Plutonium enrichment & Inner/outer $16 / 21(\% \mathrm{Pu}$ fission) \\
\hline Average burn-up & $80,000 \mathrm{MWd} / \mathrm{t}$ \\
\hline Cladding material & $\mathrm{SS} 316$ \\
\hline
\end{tabular}

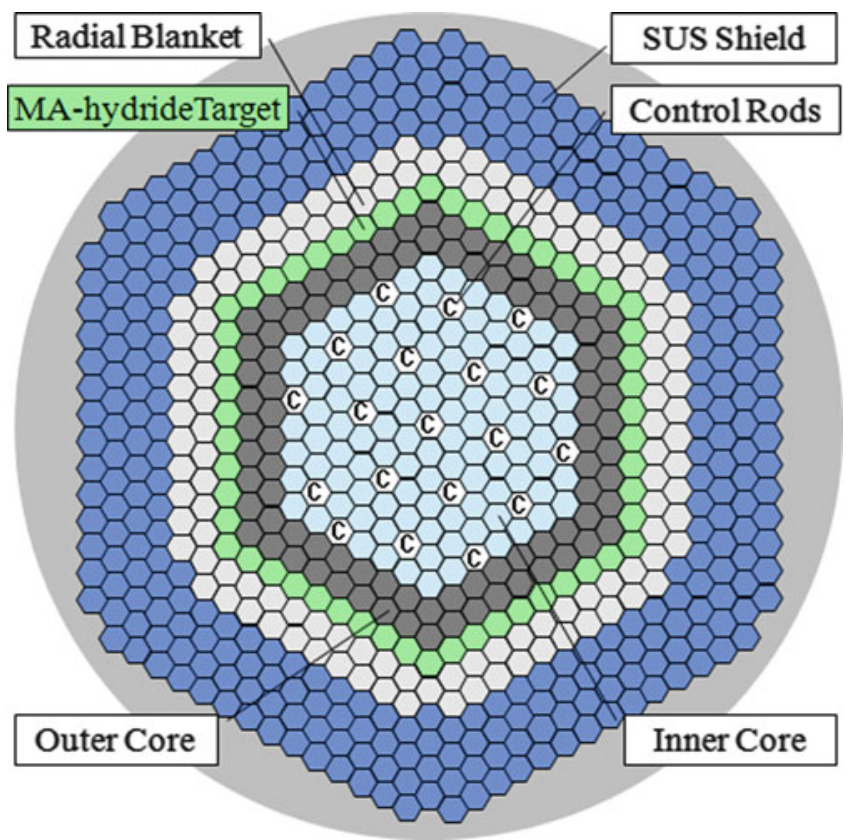

Fig. 16.3 Core layout with hydride targets

\subsection{Transmutation Calculation}

Transmutation performances of the hydride MA target and related core features in FBRs have been evaluated with the method shown in Table 16.2. A threedimensional continuous energy Monte Carlo Code MVP [9] and MVP-BURN [10] are used as burn-up calculations for evaluating the transmutation of MAs. The cross-section library applied in the calculations is JENDL-4.0, which is processed to be adjusted to the MVP code. In the burn-up calculation, the prediction-correction method is employed to improve accuracy with millions of neutron histories for the criticality calculation, where the accuracy of Eigen value is about $0.04 \%$. 
Table 16.2 Calculation method for MA transmutation

\begin{tabular}{l|l|l}
\hline Items & Methods & Notes \\
\hline $\begin{array}{l}\text { Computation } \\
\text { method }\end{array}$ & $\begin{array}{l}\text { Three-dimensional continuation } \\
\text { energy Monte Carlo analysis code; } \\
\text { MVP (burn-up routine is } \\
\text { MVP-BURN) }\end{array}$ & $\begin{array}{l}1,200,000 \text { neutron histories with } \\
120 \text { batches. Initial 20 batches are run } \\
\text { to establish the initial neutron source } \\
\text { distribution }\end{array}$ \\
\hline $\begin{array}{l}\text { Nuclear data } \\
\text { Calculation }\end{array}$ & JENDL-4.0 library & \\
\hline
\end{tabular}

Table 16.3 Comparison of reduction ratio of MAs

\begin{tabular}{l|l|l|l|l}
\hline Target & $\begin{array}{l}\text { Loading } \\
\text { mass }(\mathrm{kg})\end{array}$ & $\begin{array}{l}\text { Reduction mass } \\
(\mathrm{kg} / \text { year) }\end{array}$ & $\begin{array}{l}\text { Reduction ratio } \\
\text { after 1 year }\end{array}$ & $\begin{array}{l}\text { Effective half life } \\
\text { (year) }\end{array}$ \\
\hline $\begin{array}{l}\text { Case1: } \\
\text { MA-hydride }\end{array}$ & 335 & $91.1(33.0)^{\mathrm{a}}$ & 0.272 & 2.19 \\
\hline $\begin{array}{l}\text { Case2: } \\
\text { MA-metal }\end{array}$ & 335 & $27.6(9.4)^{\mathrm{a}}$ & 0.082 & 8.07 \\
\hline $\begin{array}{l}\text { Ratio: Case1/ } \\
\text { Case2 }\end{array}$ & 1.00 & 3.30 & 3.30 & 0.27 \\
\hline
\end{tabular}

${ }^{a}$ Values in parentheses are reduction masses by fissions

Calculations have been done for two kinds of transmutation target. In case 1, the transmutation target was the MA hydride of $\left(\mathrm{MA}_{0.1}, \mathrm{Zr}_{0.9}\right) \mathrm{H}_{1.6}$. Calculation with metal $\mathrm{MA}_{0.1} \mathrm{Zr}_{0.9}$ target without $\mathrm{H}$ was done in case 2 . The results of calculations are summarized in Table 16.3, where effective half-life is defined as the time such that the residual amount of MA is decreased to half of the MA loaded during the burn-up. The effective half-life is calculated to be 2.19 years in case 1 and 8.07 years in case 2, mainly because of the softened spectrum effect induced by the MA-hydride. The transmutation rate of the MA-hydride target is about three times higher than that of the MA-metal target. Figure 16.4 shows the change of total MA and each element of MA in the MA-hydride target with increase of time. Major elements in MA, that is, $\mathrm{Np}$ and Am are decreased simultaneously during the burnup. The contribution of long-lived $\mathrm{Cm}\left({ }^{245} \mathrm{Cm}\right.$ and $\left.{ }^{246} \mathrm{Cm}\right)$ is much smaller than that of $\mathrm{Np}$ and Am. The change of total MA in the MA-metal target is also shown in Fig. 16.4 for comparison.

The major mode of the transmutation in the present method is not fission but neutron capture (see Table 16.3). As shown in Fig. 16.5, Am and $\mathrm{Np}$ are mainly transmuted to Pu because of neutron capture, beta decay, and alpha decay. Recycled $\mathrm{Pu}$ is used as a driver fuel in this reactor. 


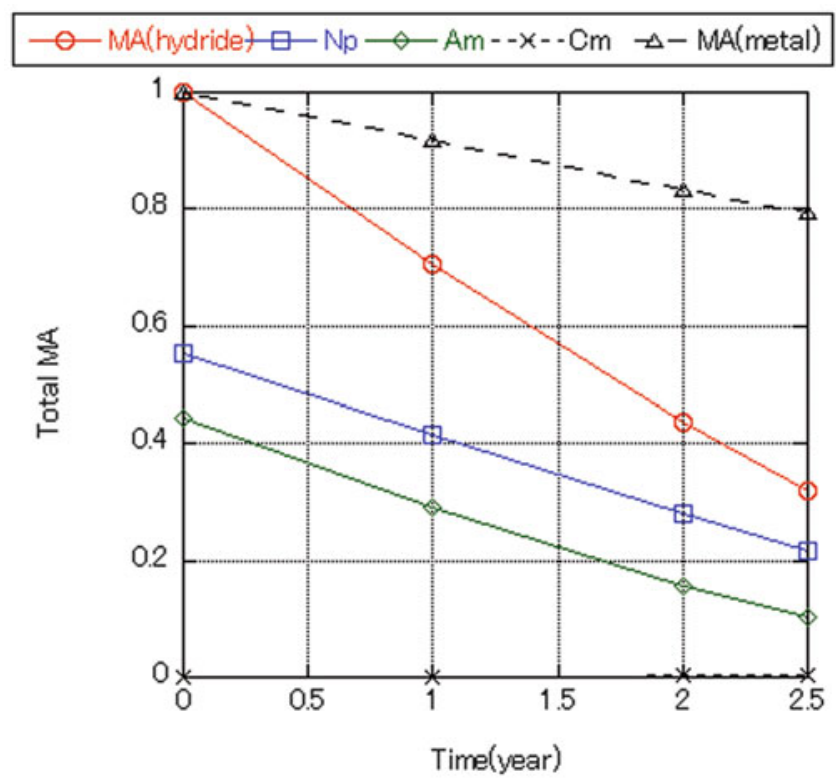

Fig. 16.4 Change of each element in MA assemblies

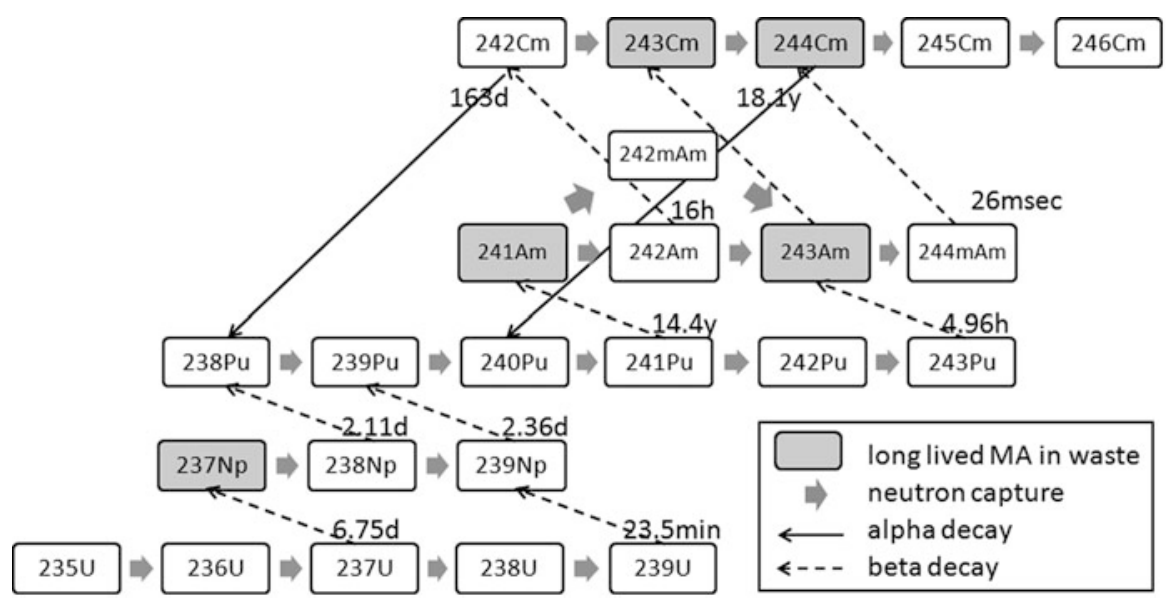

Fig. 16.5 Chain transmutations for actinide nuclides 


\subsection{Discussion}

One of the problems for hydride used in a fast spectrum core is the thermal spike wherein a large power peak occurs in the fuel pins near the hydride used zones. Figure 16.6 shows the intra-assembly radial power distribution in the outermost assembly of the core, that is, the fuel assembly adjacent to the MA-hydride assembly. If a Zr-hydride assembly without MA was used instead of the MA-hydride assembly, a large power peak appeared at the No. 15 pins adjacent to the hydride assembly (shown by pink line of $\mathrm{ZrH}_{1.6}$ case in Fig. 16.6). In our proposed case, MA-hydride works as an absorber of thermal neutrons, and thus a thermal spike is suppressed. As a result, the radial power distribution of the core has an ordinary profile in the core zones (Fig. 16.7). The power of the first row in the blanket region is, however, a little larger than that of ordinary fast reactors because of the fission reactions of MAs or daughter nuclides, although this power increase is considered to be controllable by adjusting the assembly flow distribution.

Figure 16.8 shows the mass balance of MA for the system of about three $1 \mathrm{GWe}$ class LWRs and one FBR with MA-hydride target as previously described. The LWR annually produces spent fuel with burn-up of $45 \mathrm{GWd} / \mathrm{t}$ containing $23 \mathrm{~kg}$ MA.. The mass of transmuted MA per year is almost equivalent to that produced annually in about three LWRs, which means that most of the produced MA is

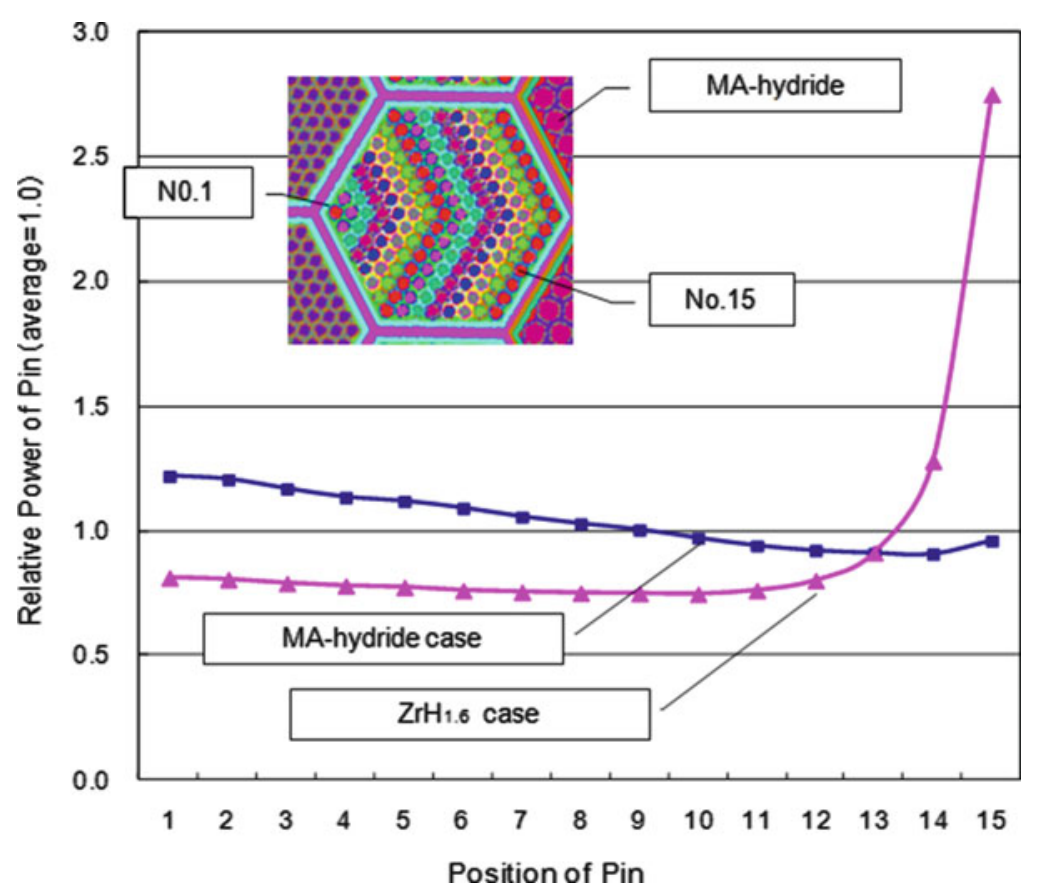

Fig. 16.6 Intra-assembly power distribution of the assembly adjacent to hydride assemblies 


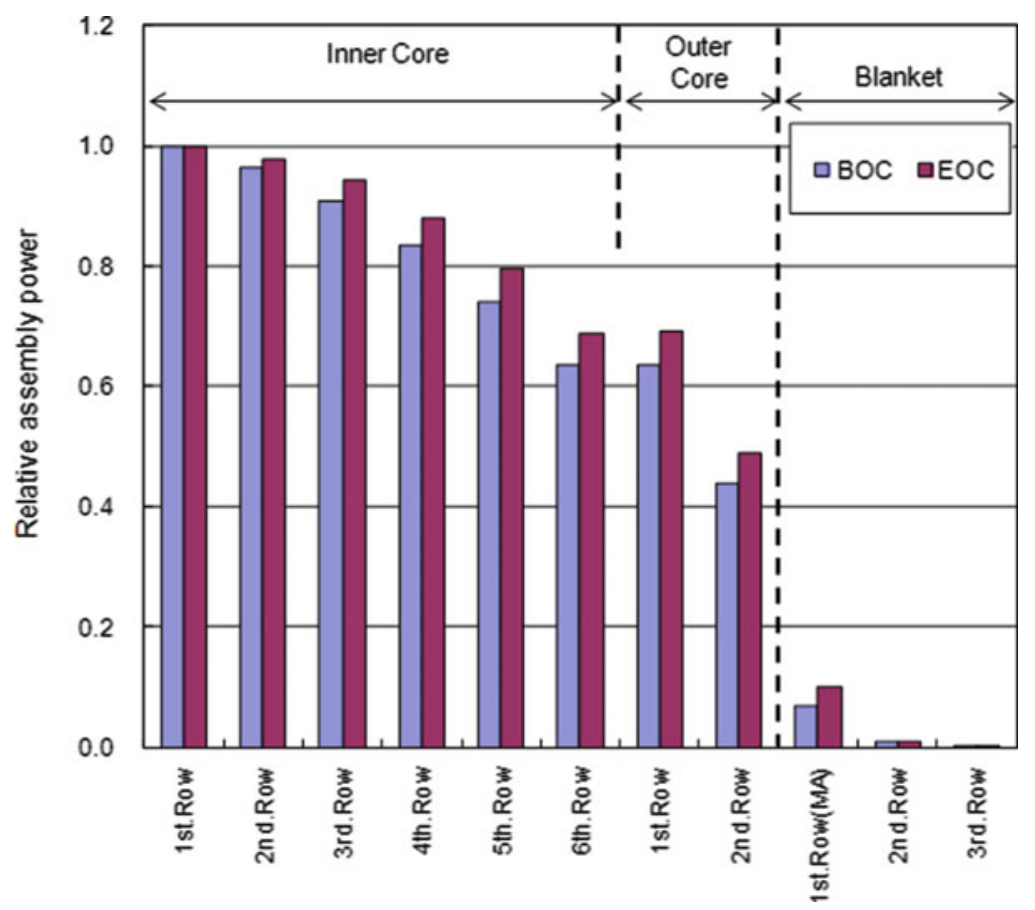

Fig. 16.7 Radial power distribution of the core

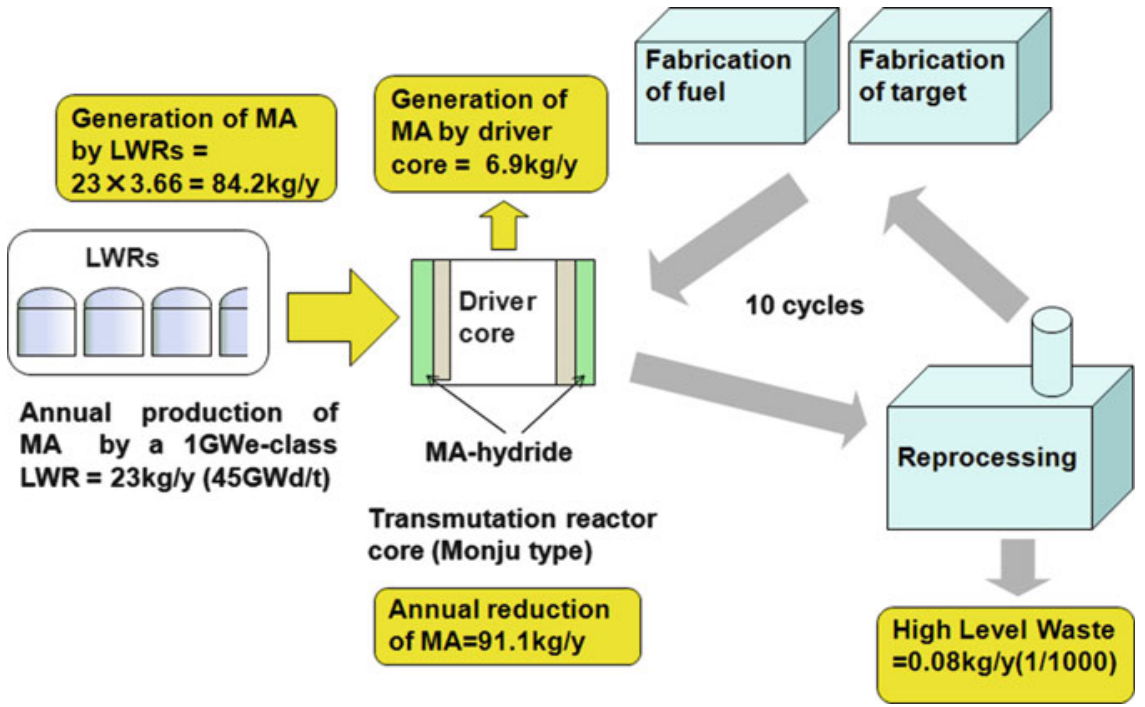

Fig. 16.8 Mass balance of MA in the system of LWRs and FBR with MA-hydride targets 
transmuted in the system and only a small amount of MA is transferred to the waste stream. As seen in Fig. 16.8, MA recycling is necessary for higher transmutation efficiency. It takes ten times the effective half-life to reduce the mass of MA to $1 / 1,000$ of the initial mass. When the irradiation time of one cycle is 2.19 year, ten fuel cycles are necessary to reduce the mass of MA to $1 / 1,000$.

\subsection{Conclusions}

Transmutation by MA-hydride targets has been studied when the hydride target assemblies are loaded in the radial blanket region of the typical fast reactor. The MA-target pin has been designed based on experience accumulated in the development of the Hf-hydride control rod. The following conclusions have been obtained.

1. The MA-hydride target shows an excellent performance for the transmutation of wastes. The effective half life is 2.19 years, which is one third of that of the MA-metal target case.

2. The MA-hydride target has advantages in lowering of peak power and increase of loading mass in the radial blanket region.

3. The transmutation mass of MA is almost equivalent to the annual production of MA from about three $1 \mathrm{GWe}$-class LWRs.

4. After MA is irradiated for 21.9 year, a mass of MA reduces to $1 / 1,000$ of that of initially loaded MA. Thus, only $1 / 1,000$ of the MA generated in a LWR should be transferred to the waste stream.

Open Access This chapter is distributed under the terms of the Creative Commons Attribution Noncommercial License, which permits any noncommercial use, distribution, and reproduction in any medium, provided the original author(s) and source are credited.

\section{References}

1. Homogeneous versus heterogeneous recycling of transuranics in fast nuclear reactors. $\mathrm{OECD/}$ NEA, Paris

2. Rome M et al (1996) Use of fast reactors to burn long-life actinides, especially Am, produced by current reactors. In: Proceedings of the Physor'96, Mito, September 16-20, 1996

3. Konashi $\mathrm{K}$ et al (1999) Transmutation of actinide nuclear wastes using hydride fuel target. Trans Am Nucl Soc 81:124-125

4. Konashi K et al (2001) Development of actinide-hydride target for transmutation of nuclear waste. In: Proceedings of the international conference on future nuclear systems (GLOBAL '01), Paris, September 9-13, 2001

5. Bates S et al (2009) Minor actinide recycle in sodium cooled fast reactors using heterogeneous targets. In: Proceedings of the meeting on advances in nuclear fuel management IV (ANFM 2009), Hilton Head Island, 12-15 April, 2009 
6. Simnad MT (1981) The U-ZrHx alloy: its properties and use in TRIGA fuel. Nucl Eng Des 64:4033-4422

7. Konashi K, Itoh K, Kido T, Kosaka Y, Seino S (2013) Development of hydride neutron absorber for fast reactor: irradiation experiment on hydride neutron absorber in BOR-60. In: Proceedings of ICAPP 2013, Jeju Island, April 14-18, 2013, paper FF232

8. Olander D, Konashi K, Yamawaki M (2012) 3.12 Uranium-zirconium hydride fuel. In: Comprehensive nuclear materials, Elsevier, pp 314-357

9. Nagaya Y, Mori T, Okumura K, Nakagawa M (2005) MVP/GMVP version 2: general purpose Monte Carlo Codes for neutron and photon transport calculations based on continuous energy and multigroup methods. JAERI 1348

10. Okumura K, Nakagawa M, Kaneko K (1997) Development of burn-up calculation code system MVP-BURN based on continuous energy Monte Carlo method and its validation (M\&C and SNA 97), vol 1. Saratoga Springs 